\title{
COMPUTATIONS OF COMPLEX EQUIVARIANT BORDISM RINGS
}

\author{
DEV PRAKASH SINHA
}

\section{Introduction}

Bordism theory is fundamental in algebraic topology and its applications. In the early sixties Conner and Floyd introduced equivariant bordism as a powerful tool in the study of transformation groups. In the late sixties, tom Dieck introduced homotopical bordism in order to refine understanding of the localization techniques employed by Atiyah, Segal and Singer in index theory. Despite the many successful computations and applications of bordism theories, equivariant bordism has been mysterious from a computational point of view, even for cyclic groups of prime order $p$ (see [11] and [12]). In this paper we present the first computations of the ring structure of the coefficients of equivariant bordism, for abelian groups. The key constructions are operations on equivariant bordism. Analogs of these operations should play an important role in equivariant stable homotopy more generally. Our main techniques involve localization and give some insight into the structure of $M U_{*}^{G}$ for a large class of groups including $p$-groups. We give a synopsis of our results now.

We denote by $M U_{*}^{G}$ the homotopical equivariant bordism ring, where $G$ is a compact Lie group. It is defined analogously to $M U_{*}$ as $\lim _{V}\left[S^{\underline{n} \oplus V}, T\left(\xi_{|V|}^{G}\right)\right]^{G}$, where $V$ ranges over isomorphism classes of complex representations of $G, S \underline{n} \oplus V$ is the one-point compactification of the Whitney sum of $\mathbb{C}^{n}$ with trivial $G$ action and $V$, and $T\left(\xi_{|V|}^{G}\right)$ is the Thom space of the universal complex $G$-bundle. In fact, we may use these Thom spaces to define an equivariant spectrum as first done by tom Dieck [7] and hence define associated equivariant homology and cohomology theories $M U_{*}^{G}(-)$ and $M U_{G}^{*}(-)$. We will carefully make these constructions in section 3 .

Euler classes play fundamental roles in our work. The Euler classes which are most important for us are those associated to a complex representation of $G$, considered as a $G$-bundle over a point. More explicitly, the Euler class associated to $V$ is a class $e_{V} \in M U_{G}^{m}(p t$.), where $m$ is the dimension of $V$ over the reals, represented by the composite $S^{0} \hookrightarrow S^{V} \rightarrow T\left(\xi_{|V|}^{G}\right)$, where the second map is "inclusion of a fiber". Euler classes multiply by the rule $e_{V} \cdot e_{W}=e_{V \oplus W}$. In homological grading $e_{V} \in M U_{-m}^{G}$, so it cannot be in the image of a geometric bordism class under the Pontrijagin-Thom map if it is non-trivial. If $V^{G}=\{0\}$ then $e_{V}$ is non-zero, reflecting the fact that $V$ has no non-zero equivariant sections. Therefore, the homotopy groups of $M U^{G}$ are not bounded below, a feature which already distinguishes it from its ordinary counterpart.

More familiar classes in $M U_{*}^{G}$ are those in the image of classes in geometric bordism under the Pontrijagin-Thom map. Given a stably complex $G$-manifold $M$, 
let $[M]$ denote the corresponding class in $M U_{*}^{G}$. Complex projective spaces give a rich collection of examples of $G$-manifolds. Given a complex representation $W$ of $G$ let $\mathbb{P}(W)$ denote the space of complex one-dimensional subspaces of $W$ with inherited $G$-action.

The starting point in our work is that after inverting Euler classes, $M U_{*}^{G}$ becomes computable by non-equivariant means. That we rely heavily on localization is not surprising because localization techniques have pervaded equivariant topology. For any compact Lie group $G$ let $R_{0}$ denote the sub-algebra of $M U_{*}^{G}$ generated by the $e_{V}$ and $[\mathbb{P}(\underline{n} \oplus V)]$ as $V$ ranges over non-trivial irreducible representations. Let $S$ be the multiplicative set in $R_{0}$ of non-trivial Euler classes. By abuse, denote the same multiplicative set in $M U_{*}^{G}$ by $S$. Then the key first result, which we emphasize is true for a large class of groups including $p$-groups, is the following.

Theorem 1.1. Let $G$ be a group such that any proper subgroup is contained in a proper normal subgroup. The inclusion of $R_{0}$ into $M U_{*}^{G}$ becomes an isomorphism after inverting $S$.

In other words, we may multiply any class in $M U_{*}^{G}$ by some Euler class to get a class in $R_{0}$ modulo the kernel of the localization map $S$. We are lead to study divisibility by Euler classes as well as the kernel of this localization map. We can do so successfully in the case when the group in question is a torus.

Let $T$ be a torus, and let $V$ be a non-trivial irreducible representation of $T$. Let $K(V)$ denote the subgroup of $T$ which acts trivially on $V$. There is a restriction homorphism (of algebras) $\operatorname{res}_{H}^{T}: M U_{*}^{T} \rightarrow M U_{*}^{H}$ for any subgroup $H$. The restriction of $e_{V}$ to $M U_{*}^{K(V)}$ is zero, as can be seen using an explicit homotopy. Remarkably, we have the following.

Theorem 1.2. The sequence

$$
0 \rightarrow M U_{*}^{T} \stackrel{\cdot e}{\rightarrow} M U_{*}^{T} \stackrel{r e s_{K(V)}^{T}}{\rightarrow} M U_{*}^{K(V)} \rightarrow 0
$$

is exact.

Note that the surjectivity of the restriction map is false for geometric bordism. One cannot for example extend the non-trivial action of $\mathbb{Z} / 2$ on two points to an $S^{1}$-action.

Using this exact sequence, we define operations which are essentially division by Euler classes. To define these operations we need to split the restriction maps. The restriction map to the trivial group is called the augmentation map $\alpha: M U_{*}^{G} \rightarrow$ $M U_{*}$. There is a canonical splitting of this map as rings which defines an $M U_{*^{-}}$ algebra structure on $M U_{*}^{G}$. All of the maps we have defined so far are in fact maps of $M U_{*}$-modules. The restriction maps to other sub-groups are not canonically split, but we do know the following from [14].

Theorem 1.3 (Comezaña). Let $G$ be abelian. Then $M U_{*}^{G}$ is a free $M U_{*}$-module concentrated in even degrees.

Hence we may fix a splitting $s_{V}$ as $M U_{*}$-modules of the restriction map $\operatorname{res}_{K(V)}^{T}$. Unless $K(V)$ is the trivial group, this splitting is non-canonical and is not a ring homomorphism.

Definition 1.4. Let $T$ and $V$ be as above. Define the $M U_{*}$-linear operation $\Gamma_{V}$ as follows. Let $x \in M U_{*}^{T}$. Then $\Gamma_{V}(x)$ is the unique class in $M U_{*}^{T}$ which satisfies

$$
e_{V} \cdot \Gamma_{V}(x)=x-s_{V}\left(\operatorname{res}_{K(V)}^{T} x\right) .
$$


For convenience, let $\beta_{V}$ denote $s_{V} \circ \operatorname{res}_{K(V)}^{T}$. We are now ready to state our main theorem.

Theorem 1.5. For any choice of splittings $s_{V}, M U_{*}^{T}$ is generated as an $M U_{*}$ algebra over the operations $\Gamma_{V}$ by the classes $e_{V}$ and $[\mathbb{P}(\underline{n} \oplus V)]$, where $n \in \mathbb{N}$ and in all instances $V$ ranges over non-trivial irreducible complex representations of $T$. Relations are as follows:

- $e_{V} \Gamma_{V}(x)=x-\beta_{V}(x)$

- $\Gamma_{V}\left(\beta_{V}(x)\right)=0$

- $\Gamma_{V}\left(e_{V}\right)=1$

- $\Gamma_{V}(x y)=\Gamma_{V}(x) y+\beta_{V}(x) \Gamma_{V}(y)+\Gamma_{V}\left(\beta_{V}(x) \beta_{V}(y)\right)$

- $\Gamma_{V} \Gamma_{W} x=\Gamma_{W} \Gamma_{V} x+\Gamma_{W} \Gamma_{V} \beta_{W}(x)-\Gamma_{W} \Gamma_{V}\left(e_{W}\right) \beta_{V}\left(\Gamma_{W} x\right)$ $-\Gamma_{W} \Gamma_{V}\left(\beta_{V}\left(e_{W}\right) \beta_{V}\left(\Gamma_{W} x\right)\right)$,

where $V$ and $W$ range over non-trivial irrreducible representations of $T$ and $x$ and $y$ are any classes in $M U_{*}^{G}$.

We may recover the structure of $M U_{*}^{G}$ for any abelian group $G$ by realizing $G$ as the kernel of an irreducible representation of some torus and using the exact sequence of Theorem 1.2.

We give both algebraic and geometric applications of our main computation. For $G=S^{1}$ and $\rho$ its standard representation, we present a geometric model of $\Gamma_{\rho}([M])$. This geometric model allows us to compute the completion map $M U_{*}^{G} \rightarrow$ $\left(M U_{*}^{G}\right)_{\hat{I}}$, where $I$ is the kernel of the augmentation map from $M U_{*}^{G}$ to $M U_{*}$. The completion theorem of Löffler, as proved by Comezaña and May, states that for $G$ abelian, $\left(M U_{*}^{G}\right)_{\hat{I}} \cong M U^{*}\left(B_{G}\right)$, where $B_{G}$ is the classifying space of $G$. So this completion map gives a connection between equivariant bordism and any equivariant theory which is defined using a Borel construction $E_{G} \times_{G}-$. We also give more classically-styled applications to the understanding of group actions on manifolds. For example, a current topic of great interest in equivariant cohomology is the investigation of $G$-manifolds with isolated fixed points, essentially extending Smith theory. We prove the following.

Theorem 1.6. Let $M$ be a stably-complex four dimensional $S^{1}$-manifold with three isolated fixed points. Then $M$ is equivariantly cobordant to $\mathbb{P}(\underline{1} \oplus V \oplus W)$ for some distinct non-trivial irreducible representations $V$ and $W$ of $S^{1}$.

The author thanks his thesis advisor, Gunnar Carlsson, for pointing him to this problem and for innumerable helpful comments. As this project has spanned a few years, the author has many people to thank for conversations which have been helpful including Botvinnik, Goodwillie, Klein, Milgram, Sadofsky, Scannell, Stevens and Weiss. He also thanks Haynes Miller for a close reading of an earlier version of this paper. Thanks also go to Greenlees, Kriz and May for sharing preprints of their work.

\section{Preliminaries}

Until otherwise noted, the group $G$ is a compact Lie group.

All $G$ actions are assumed to be continuous, and $G$-actions on manifolds are assumed to be smooth. For any $G$-space $\mathrm{X}$, we let $X^{G}$ denote the subspace of $X$ fixed under the action of $G$. The space of maps between two $G$-spaces, which we denote $\operatorname{Maps}(X, Y)$ has a $G$-action by conjugation. We denote its subspace of 
$G$-fixed maps by $\operatorname{Maps}^{G}(X, Y)$. We will often work with based spaces, in which case we assume that the basepoints are fixed by $G$. Throughout, $E_{G}$ will be a contractible space on which $G$ is acting freely. And $B_{G}$, the classifying space of $G$, is the quotient of $E_{G}$ by the action of $G$.

We will always let $V$ and $W$ be finite-dimensional complex representations of $G$. Our $G$-vector bundles will always have paracompact base spaces, so we may define a $G$-invariant inner product on the fibers. The constructions we make using such an inner product will be independent of choice of inner product up to homotopy. We will use the same notation for a $G$-bundle over a point as for the corresponding representation. We let $|V|$ denote the dimension of $V$ as a complex vector space. The sphere $S^{V}$ is the one-point compactification of $V$, based at 0 if a base point is needed. And the sphere $S(V)$ is the unit sphere in $V$ with inherited $G$-action. For a $G$-vector bundle $E$, let $T(E)$ denote its Thom space, which is the cofiber of the unit sphere bundle of $E$ included in the unit disk bundle of $E$. Thus for $V$ a representation $T(V)=S^{V}$.

Let $R^{+}(G)$ denote the monoid (under direct sum) of isomorphism classes of complex representations of $G$, and let $R(G)$ denote the associated Grothendieck ring (where multiplication is given by tensor product). We let $\operatorname{Irr}(G)$ denote the set of isomorphism classes of irreducible complex representations of $G$, and let $\operatorname{Irr}^{*}(G)$ be the subset of non-trivial irreducible representations. If $W=\sum a_{i} V_{i} \in R(G)$ where $V_{i}$ are distinct irreducible representations, let $\nu_{V}(W)$ for an irreducible $V$ be $a_{j}$ if $V$ is isomorphic to $V_{j}$ or zero if $V$ is not isomorphic to any of the $V_{i}$. Recall from the introduction that $\rho$ is the standard representation of $S^{1}$. We will by abuse use $\rho$ to denote the standard representation restricted to any subgroup of $S^{1}$. We use $\underline{n}$ or $\mathbb{C}^{n}$ to denote the trivial $n$-dimensional complex representation of a group. We will sometimes think of representations as group homomorphisms, and talk of their kernels, images, and so forth.

We rely on techniques from equivariant stable homotopy theory. Let $\Omega^{W}(X)$ denote the space of based maps from $S^{W}$ to $X$. By fixing a representation $\mathcal{U}$ with inner product, of which a countably infinite direct sum of any representation of $G$ appears as a summand, we define a $G$-spectrum $X$ to be a family of spaces $X_{V}$ indexed on subspaces of $\mathcal{U}$ equipped with $G$-homeomorphisms $X_{V} \rightarrow \Omega^{W \ominus V} X_{W}$ for all $V \subseteq W$. The basic passage to ordinary stable homotopy theory is by taking the fixed-points spectrum. Consider only subspaces $V \subset \mathcal{U}^{G}$. Then we may define the fixed-points spectrum $X^{G}$ using the family of spaces $\left(X_{V}\right)^{G}$, where the bonding maps are restrictions to fixed sets of the given bonding maps.

\section{Basic Properties of $M U^{G}$}

There are two basic definitions of bordism, geometric and homotopy theoretic. Equivariantly, these two theories are not equivalent, and we will comment on this difference later in this section.

Our main concern is the homotopy theoretic version of complex equivariant bordism, as first defined by tom Dieck [7]. Fix $\mathcal{U}$, a complex representation of which a countably infinite direct sum of any representation of $G$ appears as a summand. If there is ambiguity possible we specify the group by writing $\mathcal{U}(G)$. Let $B U^{G}(n)$ be the Grassmanian of complex $n$-dimensional linear subspaces of $\mathcal{U}$. Let $\xi_{n}^{G}$ denote the tautological complex $n$-plane bundle over $B U^{G}(n)$. As in the non-equivariant 
setting, the bundle $\xi_{n}^{G}$ over $B U^{G}(n)$ serves as a model for the universal complex $n$-plane bundle. If $V$ is a complex representation, set $\xi_{V}^{G}=\xi_{\mid}^{G} V \mid$.

Definition 3.1. We let $T U^{G}$ be the pre-spectrum, indexed on all complex subrepresentations of $\mathcal{U}$, defined by taking the $V$ th entry to be $T\left(\xi_{V}^{G}\right)$ (it suffices to define entries of a prespectrum only for complex representations). Define the bonding maps by noting that for $V \subseteq W$ in $\mathcal{U}$, letting $V^{\perp}$ denote the complement of $V$ in $W$, we have

$$
S^{V^{\perp}} \wedge T\left(\xi_{V}^{G}\right) \cong T\left(V^{\perp} \times \xi_{V}^{G}\right)
$$

Then use the classifying map

$$
V^{\perp} \times \xi_{V}^{G} \rightarrow \xi_{W}^{G}
$$

to define the corresponding map of Thom spaces. Pass to a spectrum in the usual way, so that the $V$ th de-looping is given by

$$
\lim _{W \supseteq V} \Omega^{V^{\perp}}\left(T\left(\xi_{W}^{G}\right)\right)
$$

to obtain the homotopical equivariant bordism spectrum $M U^{G}$.

From this spectrum indexed by subspaces of $\mathcal{U}$ we may pass to an $R O(G)$-graded homology theory $M U_{\star}^{G}(-)$. We will be concerned with the coefficient ring in integer gradings, which we denote $M U_{*}^{G}$. But for some arguments, we will need groups graded by complex representations of $G$, giving rise to the need for the following proposition.

Proposition 3.2. Let $V$ be a complex representation of $G$. The group $M U_{V}^{G}(X)$ is naturally isomorphic to $M U_{2|V|}^{G}(X)$.

We prove this proposition after defining the needed multiplicative structure on $M U^{G}$. The classifying map of the Whitney sum

$$
\xi_{V}^{G} \times \xi_{W}^{G} \rightarrow \xi_{V \oplus W}^{G}
$$

gives rise to a map

$$
T\left(\xi_{V}^{G}\right) \wedge T\left(\xi_{W}^{G}\right) \rightarrow T\left(\xi_{V \oplus W}^{G}\right),
$$

which defines a multiplication on $M U^{G}$. The unit element is represented by the maps $S^{V} \rightarrow T\left(\xi_{V}^{G}\right)$ induced by passing to Thom spaces the classifying map of $V$ viewed as a $G$-bundle over a point. Thus in the usual way the coefficients $M U_{\star}^{G}$ form a ring and $M U_{\star}^{G}(X)$ is a module over $M U_{\star}^{G}$.

Definition 3.3. Let $V \subset \mathcal{U}$ be of dimension n. Then the classifying map $V \rightarrow \xi_{n}^{G}$ induces a map of Thom spaces $S^{V} \rightarrow T\left(\xi_{n}^{G}\right)$, which represents an element $t_{V} \in$ $M U_{V-2 n}^{G}$ known as a Thom class.

Proof of Proposition 3.2. We show that the Thom class $t_{V}$ is invertible. The isomorphism between $M U_{V}^{G}(X)$ and $M U_{2|V|}^{G}(X)$ is then given by multiplication by this Thom class.

The class in $M U_{2 n-V}^{G}$ represented by the map $S^{2 n} \rightarrow T\left(\xi_{V}^{G}\right)$ induced by the classifying map $\mathbb{C}^{n} \rightarrow \xi_{V}^{G}$ is the multiplicative inverse of $t_{V}$. The product of this class with $t_{V}$ is homotopic to the unit map $S^{V \oplus \mathbb{C}^{n}} \rightarrow T\left(\xi_{V \oplus \mathbb{C}^{n}}^{G}\right)$.

The most pleasant way to produce classes in $M U_{*}^{G}$ is from equivariant stably almost complex manifolds. Recall that there is an real analog of $B U^{G}(n)$, which we call $B O^{G}(n)$, and which is the classifying space for all $G$-vector bundles. 
Definition 3.4. A tangentially complex $G$-manifold is a pair $(M, \tau)$ where $M$ is a smooth $G$-manifold and $\tau$ is a lift to $B U^{G}(n)$ of the map to $B O^{G}(2 n)$ which classifies $T M \times \mathbb{R}^{k}$ for some $k$.

We can define bordism equivalence in the usual way to get a geometric version of equivariant bordism.

Definition 3.5. Let $\Omega_{*}^{U, G}$ denote the ring of tangentially complex $G$-manifolds up to bordism equivalence.

Classes in geometric bordism give rise to classes in homotopical bordism through the Pontrjagin-Thom construction.

Definition 3.6. Define a map PT: $\Omega_{*}^{U, G} \rightarrow M U_{*}^{G}$ as follows. Choose a representative $M$ of a bordism class. Embed $M$ in some sphere $S^{V}$, avoiding the basepoint and so that the normal bundle $\nu$ has a complex structure. Identify the normal bundle with a tubular neighborhood of $M$ in $S^{V}$. Define PT $([M])$ as the composite

$$
S^{V} \stackrel{c}{\rightarrow} T(\nu) \stackrel{T(f)}{\rightarrow} T\left(\xi_{|\nu|}\right),
$$

where $c$ is the collapse map which is the identity on $\nu$ and sends everything outside $\nu$ to the basepoint in $T(\nu)$, and $T(f)$ is the map on Thom spaces given rise to by the classifying map $\nu \rightarrow \xi_{|\nu|}$.

The proof of the following theorem translates almost word-for-word from Thom's original proof.

Theorem 3.7. The map PT is a well-defined graded ring homomorphism.

The Pontrjagin-Thom homomorphism is not an isomorphism equivariantly as it is in the ordinary setting. A theorem of Comezaña states that $P T$ is split injective for abelian groups. The following classes illustrate the failure of the PontrijaginThom map to be an isomorphism.

Definition 3.8. Compose the map $S^{V} \rightarrow T\left(\xi_{n}^{G}\right)$, in Definition 3.3 of the Thom class with the evident inclusion $S^{0} \rightarrow S^{V}$ to get an element $e_{V} \in M U_{-2 n}^{G}$ which is called the Euler class associated to $V$.

We will see that Euler classes $e_{V}$ associated to representations $V$ such that $V^{G}=\{0\}$ are non-trivial. Thus $M U_{*}^{G}$ is not connective, a feature which already distinguishes it from $\Omega_{*}^{U, G}$ as well as $M U_{*}$. The key difference between the equivariant and ordinary settings is the lack of transversality equivariantly. For example, if $V^{G}=\{0\}$ the inclusion of $S^{0}$ into $S^{V}$ cannot be deformed equivariantly to be transverse regular to $0 \in S^{V}$.

Finally, we introduce maps relating bordism rings for different groups. Recall that ordinary homotopical bordism $M U$ can be defined using Thom spaces as in our definition of $M U^{G}$ but without any group action present.

Definition 3.9. Define the augmentation map $\alpha: M U^{G} \rightarrow M U$ by forgetting the $G$-action on $M U^{G}$. When $G$ is abelian and $H$ is a subgroup of $G$ define res ${ }_{H}^{G}$ to be the map from $M U_{*}^{G} \rightarrow M U_{*}^{H}$ by restricting the $G$-action to an $H$-action.

We need to have $G$ abelian for the map $\operatorname{res}_{H}^{G}$ to be so defined. In the abelian setting, any complex representation of $H$ extends to a complex representation of $G$, so that when its $G$-action is restricted to an $H$-action the Thom space $T\left(\xi_{n}^{G}\right)$ coincides with $T\left(\xi_{n}^{H}\right)$. 
Definition 3.10. Define the inclusion map $\iota: M U \rightarrow M U^{G}$ by composing a map $S^{n} \rightarrow T\left(\xi_{n}\right)$ with the inclusion $T\left(\xi_{n}\right) \rightarrow T\left(\xi_{n}^{G}\right)$.

On coefficients, $\iota$ defines an $M U_{*}$-algebra structure on $M U_{*}^{G}$. The kernel of $\alpha$ on coefficients is called the augmentation ideal. For example, the Euler class $e_{V}$ is in the augmentation ideal as the map $S^{0} \rightarrow S^{V}$ in its definition is null-homotopic when forgetting the $G$-action. On the other hand, $\iota$ is injective, which follows from the following proposition which is proved for example in [14].

Proposition 3.11. The composite $\alpha \circ \iota: M U \rightarrow M U$ is homotopic to the identity map.

\section{The Connection Between Taking Fixed Sets and Localization}

The connection between localization, in the commutative algebraic sense, and "taking fixed sets" has been a fruitful theme in equivariant topology. We develop this connection in the setting of bordism in this section.

The main goal of this section is to prove Theorem 1.1, which we restate here for convenience. Let $R_{0}$ denote the sub-algebra of $M U_{*}^{G}$ generated by the classes $e_{V}$ and $[\mathbb{P}(\underline{n} \oplus V)]$ as $V$ ranges over non-trivial irreducible representations. Let $S$ be the multiplicative set in $R_{0}$ of non-trivial Euler classes.

Definition 4.1. A compact Lie group $G$ is nice if every proper subgroup is contained in a proper normal subgroup.

For example, abelian groups and $p$-groups are nice.

Theorem (Restatement of Theorem 1.1). Let $G$ be a nice group. The inclusion of $R_{0}$ into $M U_{*}^{G}$ becomes an isomorphism after inverting $S$.

We prove this theorem by first explicitly computing $S^{-1} M U_{*}^{G}$ and then computing the images of generators of $R_{0}$ in $S^{-1} M U_{*}^{G}$. We start with the following lemma, which provides translation between localization and topology. For any commutative ring $R$ and element $e \in R$ let $R\left[\frac{1}{e}\right]$ denote the localization of $R$ obtained by inverting $e$.

Lemma 4.2. As rings, $\widetilde{M U_{*}^{G}}\left(S^{\oplus \infty}\right) \cong M U_{*}^{G}\left[\frac{1}{e_{V}}\right]$.

Proof. The left-hand side $\widetilde{M U_{*}^{G}}\left(S^{\oplus \infty}\right)$ is a ring because $S^{\oplus_{\infty} V}$ is an $H$-space via the equivalence

$$
S^{\oplus \infty} V \wedge S^{\oplus \infty} \cong S^{\oplus \infty} V
$$

To compute the left-hand side, apply $\widetilde{M U_{*}^{G}}$ to the identification $S^{\oplus_{\infty} V}=\underline{\lim } S^{\oplus_{n} V}$. After applying the suspension isomorphisms $\widetilde{M U_{*}^{G}}\left(S^{\oplus_{k} V}\right) \cong \widetilde{M U_{*+|V|}^{G}}\left(S^{\oplus_{k+1} V}\right)$, the maps in the resulting directed system are multiplication by the $e_{V}$.

We will see that after inverting Euler classes, equivariant bordism is computable. If $G$ is a nice group and $\left\{W_{i}\right\}$ are the non-trivial irreducible representations of $G$ then $Z=S^{\oplus_{i}\left(\oplus_{\infty} W_{i}\right)}$ has fixed sets $Z^{G}=S^{0}$ while $Z^{H}$ is contractible for any $H \subset G$. Hence, our next lemma, taken with Lemma 4.2, establishes the strong link between localization and taking fixed sets. 
Lemma 4.3. Let $X$ be a finite $G$-complex and let $Z$ be a $G$-space such that $Z^{G} \simeq$ $S^{0}$ and $Z^{H}$ is contractible for any proper subgroup of $G$. Then the restriction map

$$
\operatorname{Maps}^{G}(X, Y \wedge Z) \rightarrow \operatorname{Maps}\left(X^{G}, Y^{G}\right)
$$

is a homotopy equivalence.

Proof. The restriction map is a fibration whose fiber at a given point is the space of $G$-maps which are specified on $X^{G}$. Using the skeletal filtration of $X$, we can then filter this mapping space by spaces

$$
\operatorname{Maps}^{G}\left(D^{k} \times G / H, Y \wedge Z\right)
$$

such that the maps are specified on the boundary of $D^{k} \times G / H$, and where $H$ is a proper subgroup of $G$. A standard change-of-groups argument yields that this mapping space is homeomorphic to $\operatorname{Maps}\left(D^{k},(Y \wedge Z)^{H}\right)$, again with the map specified on the boundary. But $(Y \wedge Z)^{H}$ is contractible, and thus so are these mapping spaces. Thus, the fiber of the restriction map is contractible.

We now translate this lemma to the stable realm. For simplicity, let us suppose that our $G$-spectra are indexed over the real representation ring. We can do so by choosing specific representatives of isomorphism classes of representations. Let $K_{n} \subset K_{n+1}$ denote a sequence of representations which eventually contain all irreducible representations infinitely often and such that $K_{n}{ }^{\perp} \subset K_{n+1}$ contains precisely one copy of the trivial representation. If $G$ is finite, we can let $K_{n}$ be the direct sum of $n$ copies of the regular representation.

Definition 4.4. Let $X$ be a G-prespectrum. We define the geometric fixed sets spectrum $\Phi^{G} X$ by passing from a prespectrum $\phi^{G} X$ defined as follows. We let the entry $\left\{\phi^{G} X\right\}_{n}$ be $\left(X_{K_{n}}\right)^{G}$, the $G$-fixed set of the $K_{n}$-entry of $X$. The bonding maps are composites

$$
\left(X_{K_{n}}\right)^{G} \longrightarrow\left(\Omega^{K_{n} \perp} X_{K_{n+1}}\right)^{G} \longrightarrow \Omega^{\left(K_{n}^{\perp}\right)^{G}}\left(X_{K_{n+1}}\right)^{G}=\Omega\left(X_{K_{n+1}}\right)^{G},
$$

where the first map is a restriction of a bonding map of $X$, and the second map is restriction to fixed sets of the loop space.

While the prespectrum $\phi^{G}(X)$ depends on the choice of filtration $K_{*}$, the spectrum $\Phi^{G} X$ is independent of this choice.

Lemma 4.5. Let $Z$ be as in Lemma 4.5. Then for any G-prespectrum $X$, the prespectra $(X \wedge Z)^{G}$ and $\Phi^{G} X$ are homotopy equivalent.

Proof. From the definition of $(X \wedge Z)^{G}$, consider

$$
\left(\Omega^{W}\left(X_{W \oplus V} \wedge Z\right)\right)^{G}
$$

Applying Lemma 4.3, the restriction from this mapping space to $\Omega^{W^{G}}\left(X_{W \oplus V}\right)^{G}$ is a homotopy equivalence. Choosing $V=K_{n}$, we see that $\Omega^{W^{G}}\left(X_{W \oplus K_{n}}\right)^{G}$ is an entry of $\phi^{G} X$. The bonding maps clearly commute with these restriction to fixed sets maps, so we have an equivalence of spectra.

Note that any $Z$ as in Lemma 4.3 is an (equivariant) H-space as $Z \wedge Z \simeq Z$. Hence if $X$ is a ring spectrum so is $(X \wedge Z)^{G}$. Taking Lemma 4.2 and Lemma 4.5 together, we have the following.

Proposition 4.6. Let $G$ be a nice group and let $S$ be the multiplicative set of nontrivial Euler classes in $M U_{*}^{G}$. Then as rings $S^{-1} M U_{*}^{G} \cong\left(\Phi^{G} M U^{G}\right)_{*}$. 
To compute $\left(\Phi^{G} M U^{G}\right)_{*}$, we can use the geometry of Thom spaces. Because smashing a weak equivalence of prespectra with a complex yields another weak equivalence, we have $Z \wedge M U^{G} \simeq Z \wedge T U^{G}$ as prespectra, where $T U^{G}$ denotes the equivariant Thom prespectrum and $Z$ is as in Lemma 4.3. Hence,

$$
\Phi^{G} M U^{G} \simeq Z \wedge M U^{G} \simeq Z \wedge T U^{G} \simeq \Phi^{G} T U^{G},
$$

As required by the definition of $\Phi^{G}$, we proceed with analysis of fixed-sets of Thom spaces.

We first need the following basic fact about equivariant vector bundles.

Proposition 4.7. And let $E$ be a $G$-vector bundle over a base space with trivial $G$-action $X$. Then $E$ decomposes as a direct sum

$$
E \cong \bigoplus_{V \in \operatorname{Irr}(G)} E_{V}
$$

where $E_{V} \cong \widetilde{E} \otimes V$ for some vector bundle $\widetilde{E}$.

The following result is due to tom Dieck [7] in the case of the group $G=\mathbb{Z} / p$.

Lemma 4.8. For any compact Lie group $G$, the $G$-fixed set of the Thom space of $\xi_{n}^{G}$ is homotopy equivalent to

$$
\bigvee_{W \in R^{+}(G)_{n}} T\left(\xi_{\left|W^{G}\right|}\right) \wedge\left(\prod_{V \in \operatorname{Ir} r^{*}(G)} B U\left(\nu_{V}(W)\right)\right)_{+},
$$

where we define $R^{+}(G)_{n}$ as the subset of dimension $n$ representations in $R^{+}(G)$ and we recall that $\nu_{V}(W)$ is the greatest number $m$ such that $\oplus_{m} V$ appears as a summand of $W$.

Proof. The universality of $\xi_{n}^{G}$ implies that $\left(B U^{G}(n)\right)^{G}$ is a classifying space for $n$ dimensional complex $G$-vector bundles over trivial $G$-spaces. Using Proposition 4.7 we see that this classifying space is weakly equivalent to

$$
\coprod_{W \in R^{+}(G)}\left(\prod_{V \in \operatorname{Irr}(G)} B U\left(\nu_{V}(W)\right)\right)
$$

Over each component of this union, the universal bundle decomposes as $\xi_{1} \times \xi_{2}$, where $\xi_{1}$ is the universal vector bundle over the factor of $\prod B U(n)$ corresponding to the trivial representation. The fixed set $\xi_{1}{ }^{G}$ is all of $\xi_{1}$ while the fixed set $\xi_{2}{ }^{G}$ is the zero section. The result now follows by passing to Thom spaces.

For convenience, we define the following spectrum.

\section{Definition 4.9. Let}

$$
\mathbf{S}\left[\operatorname{Irr}^{*}(G)\right]=\underset{W \in \mathbb{Z}\left[\operatorname{Irr}^{*}(G)\right] \subset R(G)}{S^{2(|W|)} .}
$$

Define a ring spectrum structure on $\mathbf{S}\left[\operatorname{Irr}^{*}(G)\right]$ by sending the $V$ summand smashed with the $W$ summand to the $V+W$ summand.

Theorem 4.10. For any compact Lie group $G$,

$$
\Phi^{G} M U^{G} \simeq \mathbf{S}\left[\operatorname{Irr}^{*}(G)\right] \wedge M U \wedge\left(\prod_{V \in \operatorname{Ir} r^{*}(G)} B U\right)_{+} .
$$


After Lemma 4.8, the proof of this theorem is straightforward, passing from the prespectrum $\phi^{G} T U^{G}$ to the spectrum $\mathbf{S}\left[\operatorname{Irr}^{*}(G)\right] \wedge M U \wedge\left(\prod_{V \in \operatorname{Irr} r^{*}(G)} B U\right)_{+}$.

For a non-trivial irreducible representation $V$, let $f_{V}$ be the map from $\mathbb{C P}^{k}$ mapping to the $V$ th wedge summand of $\prod_{V \in \operatorname{Irr}{ }^{*}(G)} B U$ by the canonical inclusion to $B U(1) \subset B U$ on the $V$ th factor and by the trivial map on the other factors. Define $Y_{i, V}$ to be the class in the subgoup $\left.M U_{2(i-1)}\left(\prod_{V \in \operatorname{Irr} r^{*}(G)} B U\right)_{+}\right)$of $\left(\Phi^{G} M U^{G}\right)_{2 i}$ represented by $\mathbb{C P}^{i-1}$

We may now complete the central computation of this section.

Theorem 4.11. The ring $\left(\Phi^{G} M U^{G}\right)_{*}$ is a Laurent algebra tensored with a polynomial algebra as follows:

$$
\left(\Phi^{G} M U^{G}\right)_{*} \cong M U_{*}\left[e_{V}, e_{V}^{-1}, Y_{i, V}\right] .
$$

Here $V$ ranges over irreducible representations of $G, i$ ranges over the positive integers, where as indicated by notation $e_{V}$ is the image of the Euler class $e_{V} \in$ $M U_{*}^{G}$ under the canonical map to the localization and where $Y_{i, V}$ are as above.

Proof. This theorem is simple computation after Theorem 4.10. We use the computation $M U_{*}(B U) \cong M U_{*}\left[Y_{i}\right]$ as rings, where $Y_{i}$ is represented by $\mathbb{C P}^{i}$ mapping to $B U$ via its inclusion into $B U(1)$, which is standard as in [1]. Because $M U_{*}(B U)$ is a free $M U_{*}$-module, it follows from the Künneth theorem that $M U_{*}\left(\prod_{I r r^{*}(G)} B U\right)$ is a polynomial algebra as well. To finish the computation, we note that the Euler class $e_{V}$ maps to the class in of $\pi_{-|V|} \mathbf{S}\left[\operatorname{Irr}^{*}(G)\right]$ which is the generator on the $V$ th summand.

From Proposition 4.6 and the above theorem we have the following.

Corollary 4.12. $S^{-1} M U_{*}^{G} \cong M U_{*}\left[e_{V}, e_{V}^{-1}, Y_{i, V}\right]$.

We have shown the intimate relation between localization and taking fixed sets for homotopical equivariant bordism. We will also need the following geometric point of view, which dates back to Conner and Floyd.

Proposition 4.13. Let $M$ be a tangentially complex $G$-manifold. The normal bundle $\nu$ of $M^{G}$ in $M$ is a complex vector bundle.

Proof. Let $\eta$ be a complex $G$-bundle over $M$ whose underlying real bundle is $T M \times$ $\mathbb{R}^{k}$, as given by the tangential unitary $G$-structure of $M$. Then by Proposition 4.7 , $\left.\eta\right|_{M^{G}}$ decomposes as a complex $G$-bundle

$$
\left.\eta\right|_{M^{G}} \cong \eta_{1} \oplus \bigoplus_{\rho \in \operatorname{Irr}^{0}(G)} \eta_{\rho}
$$

where $\eta_{1}$ has trivial $G$-action. But we can identify $\eta_{1}$ as having underlying real bundle equal to $T M^{G} \times \mathbb{R}^{k}$. So the normal bundle $\nu$ underlies $\bigoplus_{\rho \in \operatorname{Ir} r^{0}(G)} \eta_{\rho}$, which gives $\nu$ the desired complex structure.

As Comezaña points out, this proposition would not be true if in the defintion of complex $G$-manifold we chose a complex structure on either the stable normal bundle or on $T M \times V$ for an arbitrary $V$ as opposed to $\mathbb{R}^{k}$. In these cases we could only guarantee that normal bundles to fixed sets would be stably complex. 
Definition 4.14. Let

$$
F_{*}=\bigoplus_{W \in R^{+}(G)} M U_{*-|W|}\left(\prod_{V \in \operatorname{Irr}^{*}(G)} B U\left(\nu_{W}(V)\right)\right) .
$$

Define the homomorphism $\varphi: \Omega_{*}^{U, G} \rightarrow F_{*}$ as sending a class $[M] \in \Omega_{n}^{U, G}$ to the class represented by $M^{G}$ with reference map which classifies its normal bundle.

This geometric picture of taking fixed sets of $G$-actions on manifolds fits nicely with the homotopy theoretic picture we have been developing so far.

Proposition 4.15 (tom Dieck). The following diagram commutes

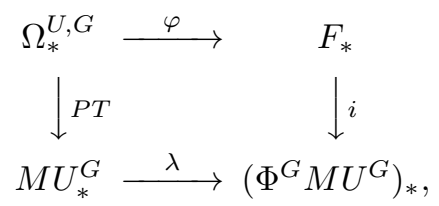

where $i$ is the inclusion map

$\bigoplus_{W \in R^{+}(G)} M U_{*-|W|}\left(\prod_{V \in I r r^{*}(G)} B U\left(\nu_{W}(V)\right)\right) \rightarrow \bigoplus_{W \in R(G)} M U_{*-|W|}\left(\prod_{I r r^{*}(G)} B U\right)$.

We may now compute the images of geometric classes in $M U_{*}^{G}$ under localization by geometric means.

Proposition 4.16. Let $V$ be an irreducible representation of $G$. The image of $[\mathbb{P}(n \oplus V)]$ in $\left(\Phi^{G} M U^{G}\right)_{*}$ is $Y_{n, V}+X$, where $X$ is $\left(e_{V^{*}}\right)^{-n}$ for one-dimensional $V$ and is zero otherwise.

Proof. We use homogeneous coordinates. There are two possible components of the fixed sets. The points whose coordinates "in $V$ " are zero, constitute a fixed $\mathbb{C P}^{n-1}$, whose normal bundle is the tautological line bundle over $\mathbb{C P}^{n-1}$ tensored with $V$. As a class in $\left(\Phi^{G} M U^{G}\right)_{*}$, this manifold with reference map to $\prod_{I r r^{*}(G)} B U$ represents $Y_{n, V}$. Alternately, when all other coordinates are zero the resulting submanifold is the space of lines in $V$, which is an isolated fixed point when $V$ is one-dimensional and is a projective space with no fixed points, as $V$ has no non-trivial invariant subspaces, when $V$ has higher dimension. As classes in $\left(\Phi^{G} M U^{G}\right)_{*}$, isolated fixed points represented negative powers of Euler classes.

We finish this section by proving that for nice groups, the inclusion of $R_{0}$ into $M U_{*}^{G}$ becomes an isomorophism after inverting Euler classes.

Proof of Theorem 1.1. Recall that from Corollary 4.12 we have that for nice groups $S^{-1} M U_{*}^{G} \cong M U_{*}\left[e_{V}, e_{V}^{-1}, Y_{i, V}\right]$. It suffices to consider the image of $S^{-1} R_{0}$ in this ring. The Euler classes and their inverses are in this image by definition. And by Proposition 1.16, the classes $Y_{i, V}$ are in this image modulo negative powers of Euler classes. 


\section{Computation of $M U_{*}^{G}$}

By Theorem 1.1, for nice groups $G$ any $x \in M U_{*}^{G}$ can be multiplied by an Euler class to get a class in $R_{0}$ modulo the annihilator of some Euler class. Our plan, which we carry out for abelian groups, is to build $M U_{*}^{G}$ from $R_{0}$ by division by Euler classes. We are faced with two questions: "when can one divide by an Euler class?" and "what are annhilators of Euler classes?" When $G$ is a torus, Theorem 1.2 answers both of these questions. Recall that $K(V)$ is the subgroup of $T$ which acts trivially on $V$.

Theorem (Restatement of Theorem 1.2). The sequence

$$
0 \rightarrow M U_{*}^{T} \stackrel{e_{Y}}{\rightarrow} M U_{*}^{T} \stackrel{r e s_{K}^{T}(V)}{\rightarrow} M U_{*}^{K(V)} \rightarrow 0
$$

is exact.

Proof. We construct the appropriate Gysin sequence and show that it breaks up into short exact sequences.

Apply ${\widetilde{M U_{T}}}^{*}$ to the cofiber sequence $S(V)_{+} \stackrel{i}{\rightarrow} S^{0} \stackrel{j}{\rightarrow} S^{V}$ to get the long exact sequence

$$
\cdots \rightarrow{\widetilde{M U_{T}}}^{2 n}\left(S^{V}\right) \stackrel{j^{*}}{\rightarrow} M U_{T}^{2 n} \stackrel{i^{*}}{\rightarrow} M U_{T}^{2 n}(S(V)) \stackrel{\delta}{\rightarrow}{\widetilde{M U_{T}}}^{2 n+1}\left(S^{V}\right) \rightarrow \cdots
$$

As $M U_{T}$ has suspension isomorphisms for any representation, ${\widetilde{M U_{T}}}^{k}\left(S^{V}\right) \cong$ $M U_{T}^{k-V}$. By Proposition 3.2, $M U_{T}^{k-V} \cong M U_{G}^{k-2}$. The map $j^{*}$ is by definition multiplication by $e_{V}$.

To compute $M U_{T}^{k}(S(V))$, we note that for a non-trivial irreducible representation of a torus $S(V)$ is homeomorphic to the orbit space $T / K(V)$. But maps from this orbit space to $M U_{T}$ are in one-to-one correspondence with maps from a single point in the orbit to the $K(V)$-fixed set of $M U_{T}$, which is homeomorphic to the $K(V)$ fixed set of $M U_{K(V)}$. We deduce that $M U_{T}^{k}(S(V)) \cong M U_{K(V)}^{k}$ and that $i^{*}$ is the restriction map.

By Comezaña's theorem (Theorem 1.3), both $M U_{T}^{*}$ and $M U_{K(V)}^{*}$ are concentrated in even degrees. Hence the long exact sequence above yields the short exact of the theorem.

Remark. Let $T=S^{1}$ and $\rho$ be the standard representation, so the restriction to the kernel of $\rho$ is the augmentation map. There is a geometric construction which reflects the fact that, by Theorem 1.2, the augmentation ideal is principal, generated by $e_{\rho}$.

Let $f: X \rightarrow Y$ be an $S^{1}$-equivariant map of based spaces which is null-homotopic upon forgetting the $S^{1}$ action. Let $F: X \times I \rightarrow Y$ be a null-homotopy. Construct an $S^{1}$-equivariant map $f_{\Sigma(F)}: X \times I \times S^{1} \rightarrow Y$ by sending

$$
(x, t, \zeta) \mapsto \zeta \cdot F\left(\zeta^{-1} \cdot x, t\right) .
$$

This map passes to the quotient

$$
X \times I \times S^{1} /\left(\left\{X \times 0 \times S^{1}\right\} \cup\left\{X \times 1 \times S^{1}\right\} \cup\left\{* \times I \times S^{1}\right\}\right),
$$

which is $S^{\rho} \wedge X$. When restricted to $S^{0} \wedge X \subset S^{\rho} \wedge X$ this map coincides with the orginal $f$, and thus gives a "quotient" of $f$ by the class $S^{0} \hookrightarrow S^{\rho}$. 
As in the introduction, fix a splitting $s_{V}$ of $\operatorname{res}_{K(V)}$ as a map of $M U_{*}$-modules. Let $\beta_{V}=s_{V} \circ \operatorname{res}_{K(V)}$. And for any $x \in M U_{n}^{T}$ let $\Gamma_{V}(x)$ be the unique class in $M U_{n+2}^{T}$ such that $e_{V} \cdot \Gamma_{V}(x)=x-\beta_{V}(x)$. The existence and uniqueness of $\Gamma_{V}(x)$ follow from Theorem 1.2 and the fact that $x-\beta_{V}(x)$ is in the kernel of $\operatorname{res}_{K(V)}$.

We are ready to prove our main theorem.

Theorem (Restatement of Theorem 1.5). For any choice of splittings $s_{V}, M U_{*}^{T}$ is generated as an $M U_{*}$-algebra over the operations $\Gamma_{V}$ by the classes $e_{V}$ and $[\mathbb{P}(\underline{n} \oplus$ $V)$ ], where $n \in \mathbb{N}$ and in all instances $V$ ranges over non-trivial irreducible complex representations of $T$. Relations are as follows:

1. $e_{V} \Gamma_{V}(x)=x-\beta_{V}(x)$

2. $\Gamma_{V}\left(\beta_{V}(x)\right)=0$

3. $\Gamma_{V}\left(e_{V}\right)=1$

4. $\Gamma_{V}(x y)=\Gamma_{V}(x) y+\beta_{V}(x) \Gamma_{V}(y)+\Gamma_{V}\left(\beta_{V}(x) \beta_{V}(y)\right)$

5. $\Gamma_{V} \Gamma_{W} x=\Gamma_{W} \Gamma_{V} x+\Gamma_{W} \Gamma_{V} \beta_{W}(x)-\Gamma_{W} \Gamma_{V}\left(e_{W}\right) \beta_{V}\left(\Gamma_{W} x\right)$

$-\Gamma_{W} \Gamma_{V}\left(\beta_{V}\left(e_{W}\right) \beta_{V}\left(\Gamma_{W} x\right)\right)$,

where $V$ and $W$ range over non-trivial irrreducible representations of $T$ and $x$ and $y$ are any classes in $M U_{*}^{G}$.

Proof. By Theorem 4.11, any class in $M U_{*}^{T}$ can be multiplied by an Euler class to give a class in $R_{0}$ modulo the kernel of the canonical map from $M U_{*}^{T}$ to $S^{-1} M U_{*}^{T}$, where $S$ is the multiplicative set of non-trivial Euler classes. By Theorem 1.2, the kernel of the map from $M U_{*}^{T}$ to the ring one obtains by inverting a single Euler class is injective, so it follows that the map to $S^{-1} M U_{*}^{T}$ is injective. Hence, any class in $M U_{*}^{T}$ is the quotient of some class in $R_{0}$ by an Euler class.

Thus, we may filter $M U_{*}^{T}$ exhaustively as

$$
R_{*}=R_{*}^{0} \subset R_{*}^{1} \subset \cdots \subset M U_{*}^{T},
$$

where $R_{*}^{i}$ is obtained from by adjoining to $R_{*}^{i-1}$ all $x \in M U_{*}^{T}$ such that $x \cdot e_{V}=$ $y \in R_{*}^{i-1}$ for some $V$. By Theorem 1.2 the set of all such $y$ for a given $V$ is $\operatorname{Ker}\left(\operatorname{res}_{K(V)}\right) \cap R_{*}^{i-1}$. The kernel of the restriction map is clearly generated by all classes $y-\beta_{V} y$. So we may obtain $R_{*}^{i}$ from $R_{*}^{i-1}$ by applying $\Gamma$ to every class in $R_{*}^{i-1}$, which proves that $M U_{*}^{T}$ is generated over the operations $\Gamma^{V}$ by $R_{0}$.

Next, we note that the relations are readily verifyable. Relation 1 holds by definition. And we may use the fact that multiplication by non-trivial Euler classes is a monomorphism to verify relations 2,3 and 1 by multiplying them by $e_{V}$, and 5 by multiplying it by $e_{V} e_{W}$.

We are left to show that the relations are complete. For convenience, if $I=$ $V_{1}, \cdots, V_{k}$ is a $k$-tuple of representations let $\Gamma_{I}(x)=\Gamma_{V_{k}} \Gamma_{V_{k-1}} \cdots \Gamma_{V_{1}} x$. We fix an ordering the representations of $T$.

We claim that a multiplicative generating set for $M U_{*}^{T}$ is given by classes $\Gamma_{I}(r)$ where $r$ is a generator of $R_{0}$ and $I$ is a (possibly empty) $k$-tuple of representations which respects the ordering we have imposed, as well as classes $\Gamma_{I}\left(\prod \beta_{V_{k}} x_{i}\right)$, where $V_{k}$ is the minimimal representation in $I$. By the relation, to construct a generating set it suffices to consider classes $\Gamma_{V}(x)$, where $x$ is either primitive itself or a product of classes in the image of $\beta_{V}$. And by relation 5 , it suffices to consider within those classes only the ones $\Gamma_{V}\left(\Gamma_{I}(x)\right)$ where $V$ is greater than any of the representations in $I$.

Next we give an additive basis for $M U_{*}^{T}$. Fix an ordering on the generators of $R_{0}$ in which $r_{i}<r_{j}$ if $r_{j}$ is in the image of some $\beta_{V}$ where $V$ is less than any $W$ such 
that $r_{i}$ is in the image of $\beta_{W}$. We define an additive basis for $M U_{*}^{T}$ in two families. Basis elements in the first family are the monomials $\Gamma_{I}\left(r_{0}\right) m$, where $m=\prod r_{i}$ is a monomial in $R_{0}, I$ respects our ordering on the representations of $T, r_{0}$ is a generator of $R_{0}$ which is not in the image of $\beta_{V_{k}}$ where $V_{k}$ is the minimal element of $I$, under our ordering $r_{0}$ is greater than any of the $r_{i}$, and for each representation $V \in I$ and each $i$ we have that $e_{V} \neq r_{i}$. Basis elements in the second family are $\Gamma_{I}\left(\prod r_{i}\right)$, where $I$ respects our ordering on the representations of $T$ and each $r_{i}$ is a generator of $R_{0}$ in the image of $\beta_{V_{k}}$ where $V_{k}$ is the minimal element of $I$.

We check that this basis is linearly independent by mapping to $S^{-1} M U_{*}^{T}$. Define a multiplicative basis of $S^{-1} M U_{*}^{T}$ using the images of elements of $R_{0}$ along with the multiplicative inverses of Euler classes. Extend our ordering of generators of $R_{0}$ to an ordering of generators of $S^{-1} M U_{*}^{T}$ in which the inverses of Euler classes are less than any generator of $R_{0}$. Now order the monomials in $S^{-1} M U_{*}^{T}$ by a dictionary ordering. Then the image of an additive basis element as defined above

$$
\prod r_{i} \cdot \prod_{V_{i} \in I} e_{V_{i}}^{-1}+\text { lower order terms. }
$$

These images are clearly linearly independent.

Finally, we show that the relations suffice to reduce any product of multiplicative generators to a linear combination of additive basis elements. Let $m$ be a monomial in the multiplicative generators defined above. If $m=e_{V} \cdot \Gamma_{I}(x) \cdots$, with $V \in I$, we may use relation 5 to express $\Gamma_{I}(x)$ as a sum of terms $\Gamma_{V}\left(\Gamma_{J}\left(x_{i}\right)\right)$ and then use relation 1 to simplify. We may thus reduce so that for each representation $V$ we do not have both $e_{V}$ and $\Gamma_{V}$ appearing in $m$. Next, note that 1 gives rise to the following relation

$$
x \Gamma_{V}(y)=\Gamma_{V}(x)\left(y-\beta_{V}(y)\right)+\beta_{V}(x) \Gamma_{V}(y) .
$$

We may use this relation repeatedly so that all of the operations which appear in $m$ are applied to a single generator of our choosing. So we reduce to terms of the form $\Gamma_{I}\left(r_{0}\right) \prod r_{i}$, where $r_{0}$ is greater than any $r_{i}$ in our ordering of generators of $R_{0}$. Finally, we use the relation 5 to reorder the representations which appear in $I$. Note that when we do so we may get terms $\Gamma_{I}\left(\prod \beta_{V}\left(x_{i}\right)\right) y$ which violate one of our conventions in that $\prod \beta_{V}\left(x_{i}\right)$ could be less than some generators which appear in $y$. We may then use equation 1 so that the operations are being applied to a maximal generator, followed by relation 5 to reorder. This process terminates. At each stage we may associate a monomial in $R_{0}$ to a product of our generators of $M U_{*}^{T}$ by forgetting all operations $\Gamma_{V}$. After an application of the relation 5 and equation 1 this associated monomial will be strictly smaller for each term than the associated monomial for the original product. Once the associated monomials are of the minimal form $\prod \beta_{V_{k}}\left(x_{i}\right)$ where $V_{k}$ is minimal among representations appearing in the indexing set for operations, we may use 4 to equate the term with an additive basis element in the second family.

\section{The Completion Map and a Construction of Conner and Floyd}

From our computations, it is clear that the structure of $M U_{*}^{G}$ is governed by the operations $\Gamma_{V}$. We call these operations Conner-Floyd operations because in the special case of $G=S^{1}, V=\rho$ the standard representation, and $[M]$ is a geometric class, there is a construction of $\Gamma_{\rho}([M])$, which dates back to work of Conner and Floyd. 
Definition 6.1. Define $\gamma(M)$ for any stably complex $S^{1}$-manifold to be the stably complex manifold

$$
\gamma(M)=M \times_{S^{1}} S^{3} \sqcup(-\bar{M}) \times \mathbb{P}(1 \oplus \rho),
$$

where $S^{3}$ has the standard Hopf $S^{1}$-action, $-\bar{M}$ is the $S^{1}$-manifold obtained from $M$ by imposing a trivial action on $M$ and taking the opposite orientation, and the $S^{1}$-action on $M \times_{S^{1}} S^{3}$ is given by

$$
\zeta \cdot\left[m, z_{1}, z_{2}\right]=\left[\zeta \cdot m, z_{1}, \zeta z_{2}\right] .
$$

Inductively define $\gamma^{i}(M)$ to be $\gamma\left(\gamma^{i-1}(M)\right)$, where $\gamma^{0}(M)=M$.

Proposition 6.2. Let $\rho$ be the standard representation of $S^{1}$. And let $M$ be a stably complex $S^{1}$-manifold. Then $\Gamma_{\rho}[M]=[\gamma(M)]$.

Proof. As the localization map is injective it suffices to check the equality in $S^{-1} M U_{*}^{S^{1}}$. By Proposition 4.15 we can compute the image of $[X],[\bar{X}]$ and $[\Gamma(X)]$ in the localization at a full set of Euler classes by computing fixed sets with normal bundle data. The result follows easily as the fixed sets of $\Gamma(X)$ are those of $X$ crossed with $\rho$ (when in the notation of equation 2 above, $m$ is fixed and $z_{2}=0$ ) along with an $\bar{X}$ crossed with $\rho$ (when $z_{1}=0$ ). In the localization, crossing with $\rho$ coincides with multiplying by $e_{\rho}^{-1}$.

This geometric construction of a single Conner-Floyd operation allows us explicit understanding of the most important representation of $M U_{*}^{T}$, namely the map from $M U_{*}^{T}$ to its completion at its augmentation ideal. As a special case of Theorem 1.2 , we know that the augmentation ideal of $M U_{*}^{S^{1}}$ is principal, generated by $e_{\rho}$. Because the augmentation map is split and multiplication by $e_{\rho}$ is a monomorphism, the completion of $M U_{*}^{S^{1}}$ at its augmentation ideal is a power series ring over $M U_{*}$ where $e_{\rho}$ maps to the power series variable under completion. As an immediate consequence of Proposition 6.2 we have the following.

Theorem 6.3. Let $[M]$ be class in $M U_{*}^{S^{1}}$ which is the image under the PontrjaginThom map of the class in geometric bordism represented by the complex $S^{1}$-manifold $M$. The image of $[M]$ under the map from $M U_{*}^{S^{1}}$ to its completion at its augmentation ideal, which is isomorphic to $M U_{*}[[x]]$, is the power series

$$
[\alpha(M)]+[\alpha(\gamma(M))] x+\left[\alpha\left(\gamma^{2}(M)\right)\right] x^{2}+\cdots,
$$

where $\alpha\left(\gamma^{i}(M)\right)$ is the manifold obtained from $\gamma^{i}(M)$ simply by forgetting the $G$ action.

Understanding this completion map for geometric classes is important for some geometric applications. As mentioned in the introduction, Comeza/ na and May have proved that for abelian $G,\left(M U_{*}^{G}\right)_{\hat{I}}(X) \cong M U_{*}\left(X \times_{G} E G\right)$. So this completion homomorphism a the connection between $M U^{G}$ and any cohomology theory which uses the Borel construction. For example, let $\epsilon$ be a genus, that is a ring homomorphism from $M U_{*}$ to some ring $E_{*}$. For an extensive introduction to genera, see [15]. We may extend $\epsilon$ to an equivariant genus $\Omega_{*}^{U, G} \rightarrow H^{*}(B G) \hat{\otimes} E_{*}$. Given a $G$-manifold $M$, take $M \times_{G} E G$ and use the genus $\epsilon$ to produce a class in $H^{*}(B G) \otimes E_{*}$ by "integration over the fiber". In our setting, for $G=S^{1}$, we 
may define this equivariant genus by taking the image of a class $[M]$ under completion, namely $f \in\left(M U_{*}^{S^{1}}\right)_{\hat{I}} \cong M U^{*}[[x]]$, and applying $\epsilon$ term-wise to get a class in $H^{*}\left(\mathbb{C P} \mathbb{P}^{\infty}\right) \hat{\otimes} E_{*} \cong E_{*}[[x]]$.

A genus $\epsilon$ is strongly multiplicative if for any fiber bundle of stably complex manifolds $F \rightarrow E \rightarrow B, \epsilon(E)=\epsilon(F) \cdot \epsilon(B)$. The following theorem is a fundamental starting point in the study of genera, saying essentially that strongly mutliplicative genera are rigid.

Theorem 6.4. Let $\epsilon$ be a strongly multiplicative genus. Then for any class $[M] \in$ $\Omega_{*}^{U, S^{1}}$, the equivariant extension $\epsilon([M])$ is equal to $\epsilon([\alpha(M)]) \in E_{*} \subset E_{*}[[x]]$.

Proof. By Theorem 6.3 the image of $[M]$ under completion is

$$
[\alpha(M)]+[\alpha(\gamma(M))] x+\left[\alpha\left(\gamma^{2}(M)\right)\right] x^{2}+\cdots .
$$

For any $X \in \Omega_{*}^{U, S^{1}}$ we have that $\epsilon([\alpha(\gamma(X))]=0$ because $\epsilon$ is strongly mulitplicative and by definition $\gamma(X)$ is the difference between a twisted product and a trivial product of $X$ and $\mathbb{C P}^{1}$.

Returning to computation of the completion map on $M U_{*}^{T}$, we now focus on Euler classes.

Proposition 6.5. The image of the Euler class $e_{\rho^{\otimes n}}$ in the completion $\left(M U_{*}^{S^{1}}\right)_{\hat{I}}$ is $[n]_{F} x$, the $n$-series in the formal group law over $M U_{*}$.

Proof. As the map from $M U_{*}^{G}$ to its completion is a map of complex-oriented equivariant cohomology theories, the Euler class of the bundle $V$ over a point gets mapped to the Euler class of $V \times{ }_{G} E_{G}$ over $B_{G}$. For $G=S^{1}, V=\rho^{\otimes n}$ the resulting bundle is the $n$ th-tensor power of the tautological bundle over $B_{S^{1}}$, whose Euler class is by definition the $n$-series.

We are now ready to state our theorem about the image of the completion map for $M U_{*}^{T}$. When $T=\left(S^{1}\right)^{k}$, the completion of $M U_{*}^{T}$ at its augmentation ideal is isomorphic to $M U_{*}\left[\left[x_{1}, \cdots x_{k}\right]\right]$.

Definition 6.6. Let $Y_{n}(x) \in M U_{*}[[x]]$ be the image of the class $[\mathbb{P}(\underline{n} \oplus \rho)]$ under the completion map.

Theorem 6.7. Let $E$ be the set of all series

$$
\left[m_{1}\right]_{F} x_{1}+{ }_{F} \cdots+{ }_{F}\left[m_{k}\right]_{F} x_{k} \in M U_{*}\left[\left[x_{1}, \cdots x_{k}\right]\right] .
$$

The image of $M U_{*}^{T}$ in its completion at the augmentation ideal is contained in the minimal sub-ring $A$ of $M U_{*}\left[\left[x_{1}, \cdots, x_{k}\right]\right]$ which satisfies the following two properties:

- $E \subset A$, and $A$ contains the series $Y_{i}(f)$ where $f \in E$ and $Y_{i}(f)$ are defined above.

- If $\alpha f \in S$ then $\alpha \in A$, for any $f \in E$.

We can recover the image of $M U_{*}^{G}$ in its completion at the augmentation ideal for general $G$ by reducing $M U_{*}\left[\left[x_{i}\right]\right]$ modulo the ideal $\left(\left[d_{i}\right]_{F} x_{i}\right)$, where $d_{i}$ are the orders of the cyclic factors of $G$. 
Proof. The first condition on $A$ says that the image contains all images of classes in $R_{0}$. Indeed, $E$ is the image of the Euler classes. And we check that the image of $\left[\mathbb{P}\left(i \oplus \rho^{\otimes n}\right)\right]$ in $\left(M U_{*}^{S^{1}}\right)_{\hat{I}}$ is $Y_{i}\left([n]_{F} x\right)$, which follows from the fact that the $S^{1}$ action on $\left[\mathbb{P}\left(i \oplus \rho^{\otimes n}\right)\right]$ is pulled back from the $S^{1}$ action on $[\mathbb{P}(i \oplus \rho)]$ by the degree $n$ homomorphism from $S^{1}$ to itself. By Theorem 4.11 we may build any class in $M U_{*}^{T}$ by dividing classes in $R_{0}$ by Euler classes. The second condition on $S$ accounts for all possible quotients by Euler classes in the image.

Suppose $f=a_{0}+a_{1} x+a_{2} x^{2}+\cdots$ is the image of $x \in M U_{*}^{S^{1}}$ under completion. Then the image of $\Gamma_{\rho}(x)$ under completion is that $a_{1}+a_{2} x+a_{3} x^{2}+\cdots$ is in the image. More generally, any $a_{i}+a_{i+1} x+\cdots$ is in the image of the completion map. So the property of a series being in the image of the completion map depends only on the tail of the series. It would be interesting to find an "analytic" way to define this image.

\section{Applichtions and Further Remarks}

In this section we give an assortment of applications and indicate directions for further inquiry.

Our first application is in answer to a question posed by Bott. Suppose a group acts on a manifold compatible with a stably complex structue and that the fixed points of the action are isolated. What can one say about the representations which appear as tangent spaces to the fixed points? If there are only two fixed points, the representations must be dual, which one can prove by Atiyah-Bott localization. What happens for three or more fixed points is an active area of inquiry in equivariant cohomology. With our bordism techniques, we can get answer some of these questions, as wel as go beyond local information.

Theorem (Restatement of Theorem 1.6). Let $M$ be a stably-complex four dimensional $S^{1}$-manifold with three isolated fixed points. Then $M$ is equivariantly cobordant to $\mathbb{P}(\underline{1} \oplus V \oplus W)$ for some distinct non-trivial irreducible representations $V$ and $W$ of $S^{1}$.

Proof. For convenience, let refer to the Euler class $e_{\rho \otimes n} \in M U_{*}^{S^{1}}$ by $e_{n}$. A complex $S^{1}$ manifold $M$ with three isolated fixed points defines a class in $M U_{*}^{S^{1}}$ whose image under $\lambda: M U_{*}^{S^{1}} \rightarrow S^{-1} M U_{*}^{S^{1}}$ is

$$
\lambda([M])=e_{a}^{-1} e_{b}^{-1}+e_{c}^{-1} e_{d}^{-1}+e_{f}^{-1} e_{g}^{-1}
$$

for some integers $a, \cdots, g$. We let $T$ denote

$$
e_{a} \cdots e_{g} \cdot \lambda[M]=e_{c} e_{d} e_{f} e_{g}+e_{a} e_{b} e_{f} e_{g}+e_{a} e_{b} e_{c} e_{d} \in M U_{*}^{S^{1}} \subset S^{-1} M U_{*}^{S^{1}} .
$$

Without loss of generality, assume $a$ is greatest of the integers $a, \cdots, g$ in absolute value. As $T$ is divisible by $e_{a}$ in $M U_{*}^{S^{1}}$, Theorem 1.2 implies that $T$ restricted to $M U_{*}^{\mathbb{Z} / a}$ must be zero. The Euler class $e_{n}$ restrict non-trivially to $M U_{*}^{\mathbb{Z} / a}$ unless $a \mid n$. Therefore one of $c, d, f, g$, say $c$ must be equal to $\pm a$. We first claim that this number must be $-a$.

Let $S_{\hat{a}}$ denote the multiplicative set generated by all the Euler classes associated to irreducible representations except for $e_{a}$. By localizing the modules in Theorem 4.11 and Theorem 1.2, we find that $S_{\hat{a}}^{-1} M U_{*}^{T}$ is generated over the operation 
$\Gamma_{a}$ by $S_{\hat{a}}^{-1} R_{0}$. Suppose that $|b|,|d|,|f|,|g|<|a|$ and that $c=a$. Then

$$
e_{a}^{-1} e_{b}^{-1}+e_{a}^{-1} e_{d}^{-1}+e_{f}^{-1} e_{g}^{-1}
$$

is in the image of the canonical map from $S_{\hat{a}}^{-1} M U_{*}^{S^{1}}$ to $S_{\hat{a}}^{-1} R_{0}$, as it is actually in the image of $\lambda$. Then we must have that $e_{b}^{-1}+e_{d}^{-1}$ is divisible by $e_{a}$ and thus is zero in $S^{-1} M U_{*}^{\mathbb{Z} / a}$ where $S$ here is the multiplicative set of all Euler classes of $\mathbb{Z} / a$. This localization of $M U_{*}^{\mathbb{Z} / a}$ is the the target of the restriction map from $S_{\hat{a}}^{-1} M U_{*}^{S^{1}}$. And by abuse we are using the same names for Euler classes for different groups. But because $|b|,|d|<|a|, e_{b}^{-1}, e_{d}^{-1}$ and their sum are non-zero in $S^{-1} M U_{*}^{\mathbb{Z} / a}$.

It is straightforward to rule out cases where some of $|b|,|d|,|f|,|g|$ are equal to $|a|$.

Next, consider the class

$$
\lambda_{\hat{a}}([M])-\lambda_{\hat{a}}\left(\left[\mathbb{P}\left(1+\rho^{\otimes a}\right)\right]\right) e_{d}^{-1} \in S_{\hat{a}}^{-1} M U_{*}^{S^{1}},
$$

where $\lambda_{\hat{a}}$ is the canonical map to this localization. Its image under the map to the full localization is

$$
e_{a}^{-1} e_{b}^{-1}-e_{a}^{-1} e_{d}^{-1}+e_{f}^{-1} e_{g}^{-1}
$$

which implies that $e_{b}^{-1}-e_{d}^{-1}$ is divisible by $e_{a}$ in $S_{\hat{a}}^{-1} M U_{*}^{S^{1}}$ or that $b \equiv d(\bmod a)$. But because $|b|,|d|<|a|$ we have that $d=a \mp b$ depending on whether $b$ is positive or negative.

Finally, as $c=-a$ and $d=b-a$ consider $\lambda\left([M]-\left[\mathbb{P}\left(1 \oplus \rho^{\otimes a} \oplus \rho^{\otimes b}\right)\right]\right)$, which will be equal $e_{f}^{-1} e_{g}^{-1}-e_{a-b}^{-1} e_{-b}^{-1}$. Case analysis of necessary divisibilities as we have been doing implies that this difference must be zero, so that the fixed-set data of $[M]$ is isomorphic to that of $\mathbb{P}\left(1 \oplus \rho^{\otimes a} \oplus \rho^{\otimes b}\right)$.

Finally, by because the localization map $\lambda$ is injective, this fixed-set data determines $[M]$ as in $S^{1}$-equivariant homotopical bordism uniquely, so that $[M]$ must equal $\left[\mathbb{P}\left(1 \oplus \rho^{\otimes a} \oplus \rho^{\otimes b}\right)\right]$ in $M U_{4}^{S^{1}}$. But a theorem of Comezaña says that the Pontrijagin-Thom map from $\Omega_{*}^{U, A}$ to $M U_{*}^{A}$ is injective for abelian groups $A$. Hence $M$ is cobordant to $\mathbb{P}\left(1 \oplus \rho^{\otimes a} \oplus \rho^{\otimes b}\right)$.

Our next application answers a question about bordism of free $\mathbb{Z} / n$-manifolds posed to us by Milgram. It is well-known that the spheres $S\left(\oplus_{k} \rho^{\otimes m}\right)$ for any $m$ relatively prime to $n$ generate $M U_{*}\left(B_{\mathbb{Z} / n}\right)$ as an $M U_{*}$-module. How are these bases related?

Theorem 7.1. Let $m$ and $n$ be relatively prime. Let $Q(x)$ be a quotient of $x$ by $[m]_{F} x$ modulo $[n]_{F} x$ in $M U_{*}[[x]]$. Define $a_{i} \in M U_{*}$ by $(Q(x))^{k}=a_{0}+a_{1} x+a_{2} x^{2}+$ ... Then

$$
\left[S\left(\oplus_{k} \rho^{\otimes m}\right)\right]=a_{0}\left[S\left(\oplus_{k} \rho\right)\right]+a_{1}\left[S\left(\oplus_{k-1} \rho\right)\right]+\cdots+a_{k-1}[S(\rho)]
$$

in $M U_{*}\left(B_{\mathbb{Z} / n}\right)$.

Proof. We use an analog of the simple fact that if $M$ is a $G$-manfold and $M \backslash M^{G}$ has a free $G$-action then $\left[\partial \nu\left(M^{G}\right)\right]=0$ in $M U_{*}\left(B_{G}\right)$, where $\partial \nu\left(M^{G}\right)$ is the boundary of a tubular neighborhood around the fixed set $M^{G}$. The null-bordism is defined by $M \backslash \nu\left(M^{G}\right)$. If the fixed points of $M$ are isolated, this will give rise to a relation among spheres with free $G$-actions. 
Let $\alpha_{0}=q^{k}$ where $q$ is a quotient of $e_{\rho}$ by $e_{\rho} \otimes m$ in $M U_{*}^{\mathbb{Z} / n}$. Inductively, let $\alpha_{i}$ be a quotient of $\alpha_{i-1}-\overline{\alpha_{i-1}}$ by $e_{\rho}$ (note that this quotient is not unique as we are working in $\mathbb{Z} / n$ equivariant bordism. Then the "fixed sets" of $\alpha_{k}$ are given by

$$
\lambda\left(\alpha_{k}\right)=e_{\rho \otimes m}{ }^{-k}-\overline{\alpha_{0}} e_{\rho}{ }^{-k}-\overline{\alpha_{1}} e_{\rho}{ }^{-k+1}-\cdots-\overline{\alpha_{k-1}} e_{\rho}{ }^{-1} .
$$

As $e_{V}^{-1}$ corresponds to a tubular neighborhood of an isolated fixed point in geometric bordism, we can deduce via transversality arguments for free $G$-actions that

$$
\left[S\left(\oplus_{k} \rho^{\otimes m}\right)\right]-\overline{\alpha_{0}}\left[S\left(\oplus_{k} \rho\right)\right]-\overline{\alpha_{1}}\left[S\left(\oplus_{k-1} \rho\right)\right]-\cdots-\overline{\alpha_{k-1}}[S(\rho)]=0
$$

in $M U_{*}\left(B_{\mathbb{Z} / n}\right)$. But the image of $\alpha_{0}$ in $\left(M U_{*}^{\mathbb{Z} / n}\right)_{\hat{I}} \cong M U_{*}[[x]] /[n]_{F} x$ is $(Q(x))^{k}$ from which we can read off that $\overline{\alpha_{i}}=a_{i}$.

Note that our expressions in $M U_{*}\left(B_{\mathbb{Z} / n}\right)$ are independent of the indeterminacy in choosing $q$ and the $\alpha_{i}$.

This old idea of using $G$-manifolds to bound and thus give insight into free $G$-manifolds has been codified by Greenlees's introduction of local cohomology to equivariant stable homotopy theory [8]. Moreover, by work of Greenlees and May, the theories we have been studying provide a unified framework in which to study the characteristic classes $E^{*}(B G)$ for any complex-oriented theory $E$. We hope that our understanding of relevant commutative algebra can lead to new insights into these techniques.

\section{REFERENCES}

[1] J.F. Adams. Stable Homotopy and Generalized Homology. University of Chicago Press, Chicago, 1974.

[2] J.F. Adams. Prerequisites (in Equivariant Stable Homotopy Theory) for Carlsson's Lecture. In Algebraic Topology, Aarhus 1982, Volume 1051 of Lecture Notes in Mathematics, Springer, Berlin, New-York, 1984.

[3] M.F. Atiyah, G.B. Segal and I. Singer. The Index of Elliptic Operators II, III. Annals of Mathematics 87 (1968), 531-578

[4] G. Carlsson. A Survey of Equivariant Stable Homotopy Theory. Topology 31 (1992), 1-27.

[5] P.E. Conner and E.E. Floyd. Differentiable Periodic Maps. Springer, Berlin-Heidelberg-New York, 1964.

[6] S. R. Costenoble and S. Waner. G-transversality revisited. Proceedings of the A.M.S. 116 (1992), 535-546.

[7] T. tom Dieck. Bordism of $G$-Manifolds and Integrality Theorems. Topology 9 (1970), 345-358.

[8] J.P.C Greenlees. $K$-homology of universal spaces and local cohomology of the representation ring. Topology 32 (1993), 295-308.

[9] J.P.C. Greenlees and J.P. May. Localization and Completion Theorems for $M U$-Module Spectra. Annals of Mathematics 146 (1997) 509-544.

[10] M. Hopkins, N. Kuhn and D. Ravenel. Morava $K$-theories of classifying spaces and generalized characters for finite groups. In Algebraic topology(San Feliu de Guixols, 1990) Volume 1509 of Lecture Notes in Mathematics Springer, Berlin, 1992.

[11] C. Kosniowski. Generators of the $\mathbb{Z} / p$ Bordism Ring, Serendipity. Mathematische Zeitschrift 149 (1976), 121-130

[12] I. Kriz. On Complex Equivariant Bordism Rings for $G=\mathbb{Z} / p$. To appear in Proceedings of Conference in Honor of Michael Boardman

[13] I. Madsen and R.J. Milgram. Classifying Spaces for Surgery and Cobordism of Manifolds. Volume 92 of Annals of Mathematics Studies. Princeton University Press, Princeton, 1979.

[14] J.P. May et. al. Equivariant Homotopy and Cohomology Theory. Volume 91 of the CBMS Regional Conference Series in Mathematics. AMS Publications, Providence, 1996.

[15] G. Segal. Elliptic cohomology (after Landweber-Stong, Ochanine, Witten, and others). Séminaire Bourbaki 1987/88. Astérisque 161-162 (1988), Exp. No. 695, 4, 187-201 (1989).

[16] D.P. Sinha. Geometric Representatives for $M U_{*}\left(B_{(\mathbb{Z} / 2)^{k}}\right)$. In preparation. 
[17] R.E. Stong. Notes on Cobordism Theory. Princeton University Press, Princeton, 1968.

[18] G. Wilson. K-Theory Invariants for Unitary G-Bordism. Quarterly Journal of Mathematics Oxford (2) 24 (1973), 499-526

Department of Mathematics, Brown University, Providence, Ri 02906

E-mail address: dps@math.brown.edu 